\title{
Lotfi A. Zadeh: A great researcher and scholar already before fuzzy sets/logic
}

\author{
Janusz Kacprzyk \\ Fellow, IEEE, IET, EurAI, IFIP, IFSA, SMIA \\ Full member, Polish Academy of Sciences \\ Member, Academia Europaea \\ Member, European Academy of Sciences and Arts \\ Member, International Academy for Systems and Cybernetic Sciences \\ Foreign member, Bulgarian Academy of Sciences \\ Foreign member, Finnish Society of Sciences and Letters \\ Foreign member, Royal Flemish Academy of Belgium for Sciences and the Arts \\ Foreign member, Spanish Royal Academy of Economic and Financial Sciences \\ Systems Research Institute Polish Academy of Sciences \\ Newelska 6, 01 - 447 Warsaw, Poland \\ e-mail: kacprzyk@ibspan.waw.pl
}

This year we have an extraordinarily important anniversary of the birth of Professor Lotfi A. Zadeh, the founder of fuzzy sets theory. The emergence of fuzzy sets and then fuzzy logic has, on the one hand, changed to a considerable extent science in the sense that, by assuming a possibility of a partial membership of an element in a set, has somehow shattered foundations so far based on the traditional "full-or-none" membership. On the other hand, fuzzy sets theory has considerably changed the life and career of many of us, now belonging to the large worldwide fuzzy sets community, because it has provided us with a fruitful research topic to work in.

In our fuzzy sets community, notably among its younger members, Lotfi, Professor Lotfi A. Zadeh, is considered to be a great researcher and scholar whose contributions have been attributed mostly to fuzzy sets and fuzzy logic, and some related topics. This is not true at all, of course, and I will try to show here the real greatness of Lotfi in a wider perspective.

First, one should understand that - as already mentioned - fuzzy sets theory, or fuzzy logic, has been a fundamental departure from the traditional way of reasoning based on the very essence of the traditional set theory, with just "full - or none" membership of an element in a set, or its 
related binary logic, with both fields having enjoyed much success for so many years, even centuries.

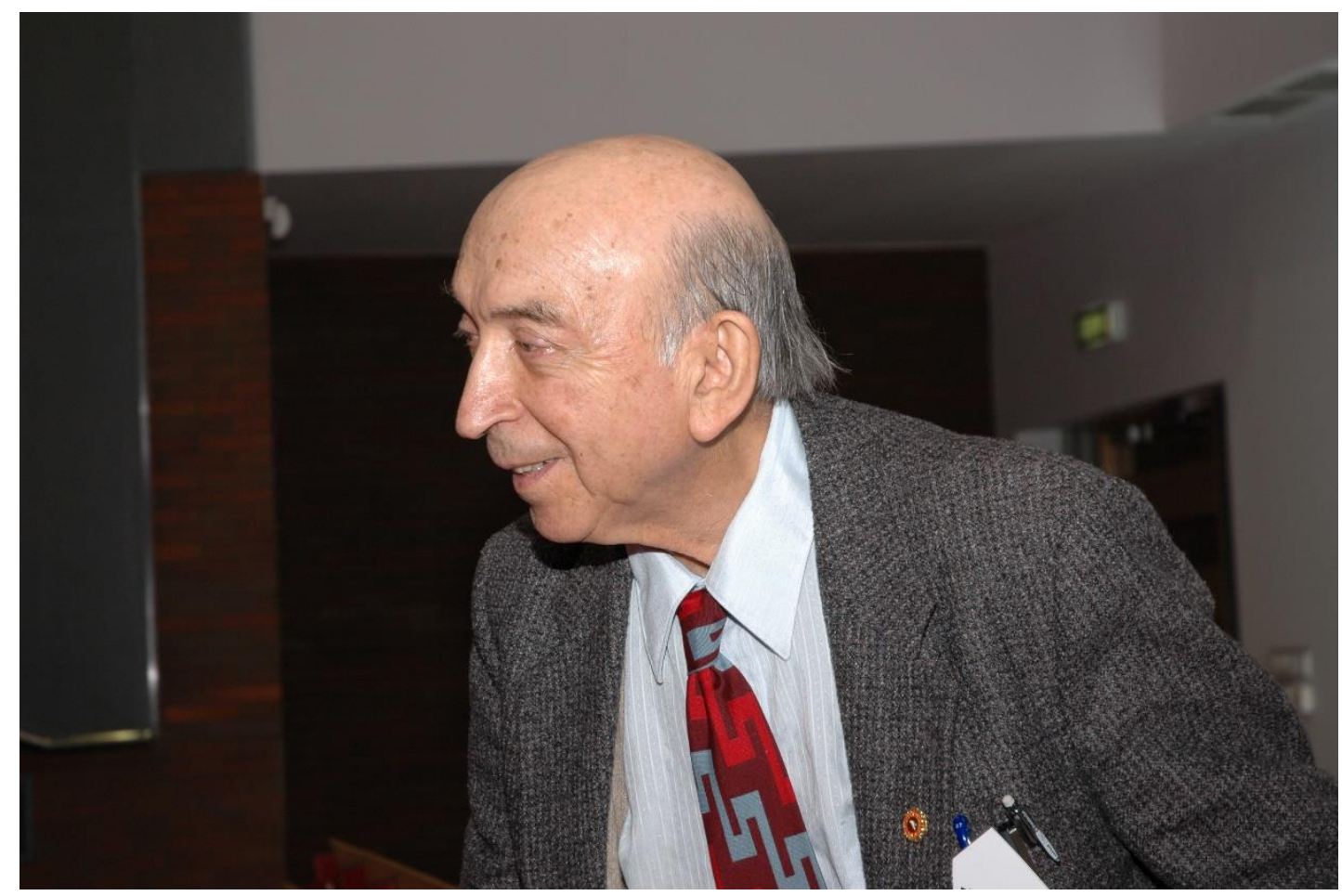

In general, it is very difficult, almost impossible to launch a new area in science that departs from what has been traditionally done and has been considered to be just obvious. Usually, such an initialization of a new area, and even a new direction, can only be done by a person who has already been famous in science. Such a person was Professor Lotfi Zadeh while proposing fuzzy sets theory. Without his worldwide scientific stature gained before fuzzy logic it would have been very difficult, most probably impossible, for fuzzy sets theory to gain its worldwide recognition it had enjoyed. Lotfi's stature has attracted in the beginning the interest of a couple of other people who have also been famous in other areas of science exemplified by Professors Richard E. Bellman (decision making and control), King-Sun Fu (pattern recognition), Hans-Juergen Zimmermann (operations research), to just mention a few. This all has helped fuzzy sets gain respect and recognition, and then enjoy an extraordinary popularity in next years.

Now, in this short note we will very briefly present Professor Zadeh's life story before 1965, the date that is attributed to the beginning of fuzzy sets, and his great research and scholarly achievements. This period in his life is crucial for the understanding of what has happened later, notably related to fuzzy sets and fuzzy logic.

The extraordinary high stature of Lotfi in science has certainly its roots in his education and illustrious academic career in early years. In 1942, he graduated from the University of Teheran with a degree in electrical engineering, and arrived at the USA with his parents in 1944. Then, he entered the famous Massachusetts Institute of Technology (MIT) as a graduate student. At that time, MIT was already one of the best universities in the world (not only in the field of technology), and it still is. Lotfi graduated from the MIT in 1946 with the M.Sc. in electrical engineering. Later, in 1949 he got his $\mathrm{PhD}$ in electrical engineering from the Columbia 
University in New York, another world famous university. His supervisor was Professor John Regazzini with whom Lotfi wrote later some great pieces of work. From 1946 to 1959 he was on the faculty of Columbia University, promoted in1957 to Full Professor, authoring many pioneering and very influential works on signal analysis, systems, control, optimization, etc. It should be noticed that the topics of his works concerned some of the most challenging topics for science and technology at that time, notably because of cold war that implied heavy financing of research that could be relevant exemplified by systems, control, etc. sciences. Then unexpectedly to many people - Lotfi left the North-East of the USA, where there have been the best universities in the USA, like MIT and Columbia University, to move to California, the University of California in Berkeley. There he continued his great works in signal analysis, systems, control, etc., thanks to which his worldwide scientific stature in these and related areas has been growing stronger and stronger. From the early 1960s, maybe even the very late 1950s, he started presenting his idea of a fuzzy set first at seminars at his university, with his famous paper published in 1965. Notice that this initial paper was published in a journal devoted to the broadly perceived area of systems and control, not mathematics, even applied.

For the purposes of this short memorial note, Lotfi's works before fuzzy sets are important and we will briefly describe them. As already mentioned, Lotfi's stature as one of great researcher and scholar has been triggered by and started during his first 10 years at Columbia University. First, by extending his results obtained in his $\mathrm{PhD}$, he co-authored, together with John Ragazzini, his supervisor, very influential papers in which they introduced what is now called the Ztransform which is commonly used in, for instance, automatic control and digital signal processing systems. Among other influential Zadeh's works one should definitely cite his novel contributions to extensions of the theory of Wiener filters, analysis of signals in the presence of noise, multiobjective optimization, optimal control, systems identification, to just mention a few.

Maybe, if one takes into account the number of works by other people, and in various areas of science and technology, both theoretical and applied, which use ideas and concepts proposed by Lotfi, then his most relevant contribution would be the state space approach he introduced together with Charles A. Desoer, his younger colleague from both first the MIT and then the University of California, Berkeley, which they joined at more or less the same time, the very end of the 1950s. The book on the linear systems and the state space approach, published in 1963 has become a „bible” of its field. It has somehow reconciled the input-output approach with the state space approach and triggered a huge research effort, both in foundations and applications in many areas. The state space approach has been used in all kinds of systems modeling, control, decision making, to just name a few fields.

The contributions of Lotfi in the pre-fuzzy period have been so diverse and theoretically relevant, and - due to space limitation - cannot be fully listed here. An interesting account of how important his contribution, and even legacy, had been is here what many famous people said during their farewell talks at various ceremonies after his passing away, or earlier, at his anniversaries. Just to mention a few, his works on, and support for the use of mathematical programming, in particular in infinitely dimensional spaces, in optimal control, notably related to the maximum principle, as well as his attempts to unify the linear systems theory and automata theory, hence bringing together electrical/systems engineering and computer sciences, are just 
examples of what many of his students and peers have mentioned about his vision and inspiration for various entire research fields.

In the early 1960, maybe even in the very late 1950s, Zadeh began to be more and more convinced that a natural tendency is obviously to deal with more and more complex and sophisticated systems, which is practically related to the fact that such systems are real world systems in which the human being is a key element. This implies that tools and techniques for the analysis of such systems should be somehow tailored to this specific features of complex human centric systems. Basically, these tools and techniques, maybe even paradigms and approaches, should more explicitly reflect the very essence of human capabilities and faculties. In 1962, in his paper groundbreaking paper: L.A. Zadeh, „From Circuit Theory to System Theory", Proceedings of the IRE, Vol. 50, 1962, pp. 856-865, he wrote:

"In fact, there is a fairly wide gap between what might be regarded as "animate" system theorists and "inanimate" system theorists at the present time, and it is not at all certain that this gap will be narrowed, much less closed, in the near future...

There are some who feel that this gap reflects the fundamental inadequacy of the conventional mathematics - the mathematics of precisely-defined points, functions, sets, probability measures, etc. - for coping with the analysis of biological systems, and that to deal effectively with such systems, which are generally orders of magnitude more complex than man-made systems... we need a radically different kind of mathematics, the mathematics of fuzzy or cloudy quantities which are not describable in terms of probability distributions...".

One should take into account that these statements were revolutionary, not to be expected by one of the most famous, and best known and established representative of systems and control sciences. Moreover, the paper was published in a prestigious journal, proceedings of the Institute of Radio Engineers (IRE), a top professional organization founded in 1912 which in 1962 merged with another well influential professional organization, the American Institute of Electrical Engineers (AIEE), to form the Institute of Electrical and Electronics Engineers (IEEE) which had since then become the largest and world's most influential professional organization in the field. Of course, Zadeh's extremely high scientific stature even at that time was for sure a key argument for the decision to publish this paper with such revolutionary ideas in an otherwise "traditional" journal.

This 1962 Lotfi's paper was practically a manifesto for the fuzzy sets and fuzzy logic... 